Canadian Oncology

Nursing Journal

Revue canadienne

de soins infirmiers

en oncologie

Volume 28, Issue 1 • Winter 2018

elSSN: 2368-8076 


\title{
Combler le fossé entre les soins oncologiques et communautaires grâce à une évaluation pancanadienne des pratiques en vigueur
}

\author{
par Danielle M. Wittal
}

\section{RÉSUMÉ}

Étant donné le nombre croissant de survivants du cancer, il devient impératif d'évaluer les soins de suivi pour bien répondre aux besoins des patients. Une évaluation des pratiques a donc été réalisée, à l'échelle provinciale et nationale, pour mieux comprendre les soins aux survivants, noter les tendances et signaler les disparités observées pendant la transition du milieu oncologique vers les soins communautaires. Des entrevues téléphoniques ont permis de recueillir les données de 9 provinces sur 10. Les documents pertinents ont également été rassemblés et les domaines de bonnes pratiques, recensés. Les façons de faire variaient grandement d'une province à l'autre et l'évaluation a permis de formuler certaines recommandations quant à l'amélioration des soins. Les résultats obtenus montrent l'importance d'encourager les patients à s'autogérer et à faire valoir leurs besoins et leurs droits. L'étude en vient à la conclusion que la survie au cancer est un sujet complexe qui nécessite communication et coordination entre les services pour assurer une transition des soins sans heurts.

\section{INTRODUCTION}

T a prévalence du cancer augmente de $3 \%$ par année en LColombie-Britannique seulement (British Columbia Cancer Agency, 2015). Au Canada, on estime que deux personnes sur cinq recevront un diagnostic de cancer dans leur vie (Société canadienne du cancer, 2015). Les statistiques laissent présager que le nombre de survivants de la maladie en Colombie-Britannique seulement passera à 250000 en 2020. Enfin, à l'échelle du pays, 196900 Canadiens ont reçu un diagnostic de cancer en 2015 (Société canadienne du cancer, 2015). On s'attend à ce que le nombre de survivants du cancer dans le monde atteigne environ 22,4 millions (Institut national du cancer, cité dans Grunfeld, 2006). Sans une transformation substantielle de la culture en place, le système de santé actuel n'arrivera pas à maintenir l'accès aux soins et la qualité du suivi offert aux patients. Les pratiques misent de plus en plus sur l'autogestion et la capacité du patient à faire valoir

\section{AU SUJET DE L'AUTEURE}

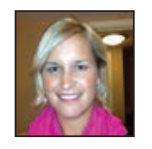

Danielle M. Wittal (chercheuse principale), Technologue en radiothérapie, Maîtrise ès sciences (M.Sc.), British Columbia

Cancer Agency, Centre for the Southern Interior, Kelowna,

Colombie-Britannique, 2118 Crosshill Drive, Kamloops (C.-B.) V1S 0C6

Danielle.wittal@bccancer.bc.ca; dwwittal@gmail.com

Tél : 778-220-8822

DOI: $10.5737 / 236880762814653$ ses besoins (notamment en renforçant le concept de soi, la confiance en soi et l'estime de soi, en stimulant la responsabilisation des patients, en favorisant un sentiment de contrôle et en aidant les patients à s'adapter à la vie avec le cancer), et maints bienfaits en découlent pour les survivants (Hagan et Donovan, 2012; Wiljer et al., 2013). Toutefois, aucun contexte politique, soit-il local ou national, n'encadre le soin des survivants au Canada à l'heure actuelle.

\section{BUT DE L'ÉTUDE}

Le présent projet avait pour but de cerner les secteurs d'intervention prioritaires dans le cadre d'une étude plus vaste qui s'aligne sur les priorités stratégiques du programme provincial de survie au cancer et de soins primaires (Provincial Survivorship and Primary Care Program) de la British Columbia Cancer Agency. L'évaluation des pratiques a permis de comprendre les forces et les faiblesses du processus de transfert des soins partout au pays et de suggérer de nouvelles mesures pour faciliter la transition des soins vers les milieux communautaires de la Colombie-Britannique. Le présent article se veut un aperçu des pratiques de transition au Canada. Nous espérons qu'il contribuera à orienter les initiatives locales et à trouver des solutions, fondées sur des données probantes, pour garantir des soins du cancer de grande qualité.

\section{MÉTHODOLOGIE}

\section{Contexte}

L'évaluation des pratiques en vigueur dans les provinces canadiennes a été réalisée entre mai 2015 et septembre 2015; 9 des 10 provinces y ont participé. Le projet comportait deux volets : une recension des écrits et une série d'entrevues.

\section{Collecte des données}

Lévaluation du milieu visait à prendre le pouls de la situation, tant à l'échelle nationale que provinciale, et à déterminer les activités, les événements, les tendances, les enjeux et les attentes propres à la transition des soins oncologiques vers le milieu communautaire. Lévaluation a comporté 1) une recension des écrits et 2) une série d'entrevues avec des personnes de partout au pays assumant, notamment, les fonctions suivantes : chefs de file en recherche sur les survivants, infirmière conseillère en oncologie, directeur des soins de soutien, gestionnaire de programme à l'intention des survivants, experts en intégration des soins axés sur la personne et intervenants-pivots pour les soins aux patients. L'étude prend la forme d'une analyse thématique. Après de nombreuses lectures 
attentives du corpus, cette approche a été jugée la plus adaptée pour relever les thèmes généraux abordés dans les documents et les entrevues (Daly, Kellehear et Gliksman, cité dans Fereday et Muir-Cochrane, 2006).

Comme l'étude faisait appel à des savoirs codifiés et à des savoirs tacites tout en employant une approche passive de la collecte de données, il n'était pas nécessaire d'obtenir le feu vert de la part des instances locales d'éthique ou des structures de gouvernance. L'Université Sheffield Hallam a approuvé l'étude, étant donné qu'elle s'inscrit dans un programme d'études supérieures. En outre, la participation aux entrevues téléphoniques supposait un consentement éclairé. Les participants n'ont subi aucun préjudice physique, psychologique ou émotionnel et la confidentialité de tous a été respectée.

\section{Stratégie de recherche pour la recension des écrits}

La recherche s'est faite dans les interfaces Ovid et EBSCOhost afin d'incorporer les bases de données CINAHL avec textes intégraux, MEDLINE avec textes intégraux, PubMed-NCBI, EBM (Cochrane), EMBASE et PsycInfo.

Voici les termes recherchés : transition des soins ou transition, oncologie, continuité des soins, organisation et administration, survie, communication, médecin omnipraticien, rôle du médecin, relation patient-médecin, habitudes de pratique des médecins, besoins et exigences des services de santé, médecins et psychologie, soins des patients, survivants du cancer ou survivants, tumeurs, suivi, plans de soins, Canada, Colombie-Britannique, Alberta, Saskatchewan, Manitoba, Ontario, Québec, Terre-Neuve, Île-du-Prince-Édouard, Nouvelle-Écosse, Nouveau-Brunswick.

Critères d'inclusion : La recherche initiale portait sur les études datées du 1er janvier 2005 au 31 décembre 2015; toutefois, des articles publiés avant 2005 (jusqu’à 1993) ont été inclus; données publiées; documentation parallèle.

Critères d'exclusion : pédiatrique ou enfants, archives Medline.

Au départ, la recherche ne retenait que les articles canadiens, mais des précisions se sont avérées nécessaires, alors la recherche a été étendue, ce qui a permis de multiplier les références, mais aussi d'avoir un aperçu de la situation à l'échelle mondiale.

La recension des écrits a permis de cerner des thèmes qui ont ensuite pu être étoffés en analysant les entrevues : rôles et responsabilités; mode et moment de transmission de l'information; modèles de soins. Bien délimiter qui est responsable de quel soin pour le patient et pouvoir transmettre la bonne information au bon moment sont essentiels pour habiliter le médecin offrant des soins primaires à assumer la responsabilité exclusive des soins de suivi. En implantant un modèle de soin approprié et adapté au mieux à chaque région du Canada, et en recourant dans la pratique à un plan de survie, des solutions réalistes pourront être proposées pour répondre aux besoins soulignés ici.

\section{Participants à l'entrevue}

Les participants ciblés pour les entrevues étaient les chefs de file en recherche sur les survivants, les infirmières conseillères en oncologie, les directeurs des soins de soutien, les gestionnaires de programme à l'intention des survivants, les experts en intégration des soins axés sur la personne et les intervenants-pivots pour les soins aux patients. Les ressources du programme provincial de survie au cancer et de soins primaires de la British Columbia Cancer Agency, le Partenariat canadien contre le cancer et une recherche sur Internet ont permis de trouver une personne-ressource à contacter dans chaque province. Chacune d'elle a reçu un courriel lui expliquant les visées du projet et lui proposant de prendre rendez-vous pour une entrevue. Lorsque la personne contactée en premier lieu n'était pas la mieux placée pour discuter du sujet à l'étude, elle suggérait une autre ressource. Dans bien des cas, il a fallu communiquer avec trois ou quatre personnes différentes par province pour trouver le participant idéal.

Les entrevues ont été réalisées par la chercheuse principale à partir d'une série de questions (voir le tableau 1). Des notes d'observation ont également été consignées. Après l'entrevue initiale, d'autres discussions ont eu lieu par téléphone ou par courriel pour obtenir des éclaircissements ou de la documentation supplémentaire, le cas échéant. Les documents pertinents ont été fournis par chacune des provinces; ils comprenaient les avis de congé ou de transfert des soins, la trousse d'information remise au moment du congé, les plans de soins des survivants et les aide-mémoire à l'intention des patients et des médecins de première ligne rappelant les recommandations en matière de suivi. Les documents ont été inclus uniquement en guise de référence pour orienter et inspirer le changement au Canada.

\section{Tableau 1 : Guide de questions pour les entrevues}

\section{Questions à poser pour l'évaluation des pratiques}

1. Quelle est la pratique en vigueur pour la transition des soins vers le milieu communautaire des patients en oncologie qui ont terminé leur traitement actif? Est-ce l'oncologue ou le médecin de première ligne qui est en charge des soins primaires? Quelle information le patient et le fournisseur de soins primaires reçoivent-ils au moment de la transition?

2. Existe-t-il des normes provinciales encadrant le processus et les objectifs de la transition des soins?

3. Avez-vous organisé des groupes de discussion, des séances de consultation ou un projet pilote pour transmettre les renseignements nécessaires au sujet de la transition des soins? Les médecins de première ligne et les oncologues ont-ils participé à la démarche?

4. Si vous utilisez un plan de soins pour les survivants, quelle forme prend-il? Quels renseignements fournit-il au patient et au fournisseur de soins primaires?

5. Qui se charge de transférer les soins aux médecins de première ligne? L'information est-elle transmise de manière informelle ou dans le cadre d'une réunion officielle? Si oui, qui y participe?

6. Si vous utilisez un plan de soins pour les survivants, s'agit-il d'un document papier, électronique ou les deux? 


\section{RÉSULTATS}

\section{Variations dans les pratiques d'attribution du congé}

Les normes de pratique définissant la transition des soins de l'oncologie vers le milieu communautaire varient grandement d'une province à l'autre. En général, le suivi des survivants du cancer n'est pas encadré par des normes ou lignes directrices provinciales, car il n'y a pas de consensus parmi les oncologues. Exception faite du Manitoba, les pratiques varient grandement d'un site à l'autre pour les soins de suivi, selon l'oncologue traitant et le type de cancer. Dans chaque province, il existe tout de même un moyen de communication entre l'oncologue et le médecin de première ligne, notamment sous forme d'un avis de congé dicté ou d'un avis de transfert des soins envoyé par courriel ou par la poste. Toutefois, l'information transmise n'est généralement pas standardisée. Dans toutes les provinces, c'est l'oncologue qui se charge de signer le congé des patients et de remettre la responsabilité des soins au médecin de première ligne.

\section{Exemples de bonnes pratiques}

Aux fins de la présente étude, les bonnes pratiques désignent les initiatives en place ou les documents conçus pour faciliter le processus de transition des soins oncologiques vers le milieu communautaire. Interrogés sur les lacunes dans les soins de transition, notamment au niveau des rôles et responsabilités, du mode et du moment de transmission de l'information, et des modèles de soins, les participants aux entrevues ont repris sans ambages les mêmes thèmes déjà relevés dans la littérature. Les exemples de bonnes pratiques glanées au fil des entrevues et décrites ci-après contribueront peut-être à combler les lacunes dans les domaines ciblés.

\section{Aide-mémoire}

En Alberta, un aide-mémoire à l'intention des patients et des médecins de première ligne permet de mieux outiller ces derniers afin qu'ils se sentent à l'aise de surveiller les signes de récidive, quels que soient les types de cancer. La procédure décrite dans l'aide-mémoire prévoit un examen clinique tous les 6 mois pendant les deux premières années, puis un examen par an pendant les 3 à 5 années suivantes; elle recommande également un examen diagnostique tous les ans pendant cinq ans. Le médecin peut noter la date de l'examen et ses observations directement sur l'aide-mémoire, ce qui permet au patient de savoir quand auront lieu les rendez-vous et au médecin d'adopter de meilleures pratiques de suivi.

En Nouvelle-Écosse, les médecins de première ligne utilisent des notes de rétroaction, qui sont jointes à l'avis de transfert des soins. Cet excellent outil permet aux médecins de première ligne de s'exprimer et de donner leur opinion sur l'information reçue et exigée au moment de la transition. Il est conçu pour aider les médecins de première ligne à soigner efficacement les patients atteints de cancer. L'aide-mémoire des soins de suivi à l'intention des patients et des médecins de première ligne et les notes de rétroaction pourraient être adoptés ailleurs afin de mieux arrimer les soins en oncologie et les soins communautaires.

\section{Rendez-vous de transition}

Au Manitoba, à l'heure actuelle, la transition des soins est systématiquement intégrée à la pratique clinique et se fait pendant un rendez-vous réservé à cette fin. Lorsque le patient a terminé les traitements et qu'il entre en phase de suivi, la clinique fixe un rendez-vous de transition. Que le fournisseur de soins principal demeure le même ou non, le rendez-vous de transition a toujours lieu. C'est l'oncologue qui est responsable du rendez-vous; il évalue la détresse du patient, l'aiguille vers les services appropriés et lui remet un document résumant le traitement et le plan de soins personnalisé (une copie est envoyée au médecin de première ligne et au chirurgien). Le rendez-vous de transition succède aux premiers examens de suivi (un tomodensitogramme pour le cancer colorectal et une mammographie pour le cancer du sein). Il a généralement lieu entre 6 semaines et 3 mois après la fin du traitement actif. Pendant ce rendez-vous, le patient reçoit le plan de soins individualisé à conserver dans ses dossiers; une copie est envoyée par télécopieur au médecin de première ligne.

En Ontario, les pratiques en vigueur varient d'un centre de traitement régional à l'autre; toutefois, pour les survivants du cancer du sein et du cancer colorectal, la transition des soins emprunte l'une des trois voies suivantes : 1) les patients sont confiés directement à un médecin de première ligne, 2) les patients consultent une infirmière praticienne, une infirmière en pratique avancée ou un oncologue en clinique de transition, qu'ils verront 1 à 3 fois avant d'être ensuite transférés à leur médecin de première ligne ou 3) les patients reçoivent des soins partagés, autrement dit, ils verront à la fois leur médecin de première ligne et l'oncologue, le radio-oncologue ou le chirurgien-oncologue pendant une période de temps déterminée. L'information fournie au patient et au médecin de première ligne varie également entre les régions, mais dans tous les cas, le médecin remet au patient un résumé du plan de soin ou du traitement, sous une forme ou sous une autre. Lorsque la région dispose d'une clinique de transition, les patients y reçoivent un rendez-vous. Ce rendez-vous sera l'occasion pour eux de se faire expliquer leur plan de soins et d'obtenir des réponses aux questions toujours en suspens. En fin de compte, c'est l'oncologue qui doit déterminer si le patient est prêt pour la transition des soins. La procédure et les fournisseurs de soins concernés varient selon les régions et le modèle adopté dans une région donnée.

\section{Coordonnateur de la transition des soins}

La Saskatchewan Cancer Agency travaille actuellement à un projet de création d'un poste de coordonnateur de la transition des soins. Ce programme de coordination s'inspire d'un programme de navigation des patients dont le responsable idéal serait travailleur social. Le coordonnateur de la transition des soins a pour rôle d'aider les patients à gérer leurs soins en leur procurant de l'information sur les programmes communautaires et les ressources existantes dans la région ou le secteur où ils vivent. Le coordonnateur de la transition s'occupera de cerner et d'évaluer la capacité et les exigences du patient et de sa famille afin de conjuguer les services et de répondre à leurs besoins en soins de soutien. Il communiquera avec les patients dans les deux semaines suivant leur congé des soins oncologiques. Il réalisera une évaluation par téléphone, ce qui lui permettra d’apaiser 
Tableau 2 : Résumé de l'évaluation pancanadienne

\begin{tabular}{|c|c|c|c|}
\hline Province & Pratique actuelle en matière de transition & Recherche en cours & Plan de soins pour les survivants \\
\hline Alberta & $\begin{array}{l}\text { Aucune norme provinciale; les pratiques varient selon } \\
\text { le type de cancer, l'oncologue traitant et le secteur } \\
\text { géographique. L'oncologue est responsable de la transition. } \\
\text { Le patient et le médecin de première ligne reçoivent } \\
\text { chacun une copie de l'avis de transfert des soins; l'avis est } \\
\text { standardisé. }\end{array}$ & $\begin{array}{l}\text { Un projet pilote lancé en septembre } 2015 \\
\text { voulait mettre à l'essai une procédure } \\
\text { intégrée pour optimiser l'application des } \\
\text { nouvelles lignes directrices provinciales en } \\
\text { matière de suivi, ainsi que le recours aux } \\
\text { avis destinés au patient et au médecin de } \\
\text { première ligne, de même que les outils et } \\
\text { les ressources connexes pour les patients } \\
\text { atteints de cancer du sein au stade } \\
\text { précoce et de cancer de la prostate traité } \\
\text { par radiothérapie externe. }\end{array}$ & Pas en pratique à l'heure actuelle. \\
\hline Manitoba & $\begin{array}{l}\text { Très variables pour l'instant. Les pratiques changent } \\
\text { selon que le patient a un médecin de première ligne et } \\
\text { qu'il a établi une bonne relation avec lui. Un rendez-vous } \\
\text { en clinique de transition est prévu entre } 6 \text { semaines et } \\
3 \text { mois après la fin du traitement actif, mais on n'y a pas } \\
\text { recours systématiquement. Les patients reçoivent un plan } \\
\text { de soins personnalisé; une copie du plan est envoyée par } \\
\text { télécopieur au médecin de première ligne. }\end{array}$ & $\begin{array}{l}\text { Un projet pilote a été mené à bien; il a } \\
\text { donné lieu à des discussions informelles } \\
\text { qui ont permis de concevoir les résumés } \\
\text { de traitement et les plans de soins des } \\
\text { survivants du cancer. }\end{array}$ & $\begin{array}{l}\text { En vigueur à l'heure actuelle pour } \\
\text { les types de cancers suivants: } \\
\text { colorectal, lymphome, ovaires, } \\
\text { péritoine et trompes de Fallope. } \\
\text { La leucémie, la greffe de moelle } \\
\text { osseuse et les cancers thoraciques } \\
\text { devraient rejoindre les rangs } \\
\text { en } 2016 .\end{array}$ \\
\hline Québec & S.O. & S.O. & S.O. \\
\hline $\begin{array}{l}\text { Nouveau- } \\
\text { Brunswick }\end{array}$ & $\begin{array}{l}\text { Aucune norme provinciale; les pratiques varient d'une } \\
\text { clinique à l'autre. L'oncologue traitant est responsable de } \\
\text { la transition des soins. L'avis de congé, dont la forme n'est } \\
\text { pas standardisée, est envoyé au médecin de première ligne. }\end{array}$ & Rien présentement. & Pas en pratique à l'heure actuelle. \\
\hline $\begin{array}{l}\text { Nouvelle- } \\
\text { Écosse }\end{array}$ & $\begin{array}{l}\text { Aucune norme provinciale; les pratiques varient d'une } \\
\text { clinique à l'autre. L'avis de transfert des soins est remis } \\
\text { au patient et au médecin de première ligne, ainsi que des } \\
\text { notes dictées personnalisées. }\end{array}$ & $\begin{array}{l}\text { En 2010, une société d'experts-conseils } \\
\text { a été engagée pour organiser des groupes } \\
\text { de discussion et des entrevues avec des } \\
\text { oncologues et des médecins de première } \\
\text { ligne, ce qui a permis d'établir en partie la } \\
\text { forme et le contenu des avis de transfert } \\
\text { des soins. }\end{array}$ & $\begin{array}{l}\text { En vigueur à l'heure actuelle } \\
\text { pour le cancer colorectal, le } \\
\text { cancer du sein et le cancer de la } \\
\text { thyroïde. Les plans ne sont pas } \\
\text { personnalisés. }\end{array}$ \\
\hline $\begin{array}{l}\text { Île-du-Prince- } \\
\text { Édouard }\end{array}$ & $\begin{array}{l}\text { Aucune norme provinciale; les pratiques dépendent de } \\
\text { l'oncologue. Un avis de consultation est fourni au médecin } \\
\text { de première ligne dans les cas où il devra prendre en } \\
\text { charge le suivi. }\end{array}$ & Rien jusqu'à présent. & Pas en vigueur à l'heure actuelle. \\
\hline Terre-Neuve & $\begin{array}{l}\text { Aucune norme provinciale; les pratiques varient selon que } \\
\text { le patient reçoit un traitement adjuvant. Le patient et le } \\
\text { médecin de première ligne reçoivent le plan de soins par } \\
\text { écrit; il comprend les examens recommandés ainsi que les } \\
\text { lignes directrices pour les soins de suivi. }\end{array}$ & $\begin{array}{l}\text { Rien jusqu'à présent; toutefois, l'équipe } \\
\text { de travail social offre un atelier sur la } \\
\text { transition des soins deux fois par année. }\end{array}$ & $\begin{array}{l}\text { En vigueur à l'heure actuelle pour } \\
\text { le cancer du sein et le cancer } \\
\text { colorectal. }\end{array}$ \\
\hline
\end{tabular}


les inquiétudes des patients sur les sujets suivants : crainte de récidive, douleur chronique, fatigue mentale ou physique, lymphœdème, changements au niveau des relations ou des fonctions sexuelles, modification de l'image corporelle, besoins ou changements alimentaires, question sur les régimes d'assurance-maladie ou d'assurance-vie, problèmes financiers, enjeux liés à l'emploi, dépression et anxiété, incertitude face à l'avenir, remise en question du sens de leur vie, isolation sociale, manque de communication ou de coordination entre les fournisseurs de soins de santé.

\section{Tableau 3 : Buts et objectifs du rôle de coordonnateur de la transition des soins}

- Comprendre les rouages d'un système de santé complexe et quelque peu fragmenté au point de transition avec les ressources communautaires.

- Donner aux patients les moyens d'utiliser de manière optimale les ressources communautaires et les encourager à prendre en charge leurs soins en adoptant une approche axée sur l'information, la communication et le soutien aux décisions.

- Mettre à la disposition des patients qui terminent leur traitement et entrent en phase de survie une personne-ressource unique pour favoriser la continuité des soins.

- Le coordonnateur de la transition des soins concentre son attention sur l'aspect communautaire, c'est-à-dire qu'il travaillera avec la famille du patient, ainsi qu'avec les groupes et les organisations communautaires, pour établir des liens communautaires solides et accroître leur capacité de satisfaire aux besoins en matière de soins de suivi des survivants du cancer.

- Le soutien que recevront les patients pour renforcer et maintenir leur relation avec leur omnipraticien, particulièrement en milieu rural, ne pourra que faciliter le rôle du médecin omnipraticien ou du médecin de première ligne. Si les patients ne sont pas suivis par un omnipraticien, ils recevront de l'aide pour en trouver un.

- Tout au long de la trajectoire des soins du cancer, les besoins des patients et des collectivités où ils vivent peuvent changer; le coordonnateur de la transition des soins devra donc être réceptif, souple et avisé. En maintenant une communication constante avec les différents intervenants, il sera plus à même de cerner ces besoins changeants.

\section{Ateliers sur la survie au cancer}

La Saskatchewan propose aux patients qui ont terminé leur traitement un programme de transition offrant de l'information de base sur la survie au cancer. En Ontario, certains centres régionaux de traitement du cancer organisent des séances d'information aux survivants du cancer pendant la période de transition ou donnent de l'information personnalisée sur différents aspects de la survie au cancer (p. ex. les effets secondaires ou persistants possibles, une bonne alimentation et un mode de vie sain).

L'Île-du-Prince-Édouard offre de son côté un éventail de programmes, notamment Look Good Feel Better (un programme national qui propose des outils pour paraître et se sentir à son mieux pendant les traitements) ainsi qu'un programme sur la transition des soins du cancer (d'une durée de 3 heures, il s'adresse aux patients ayant terminé leur traitement actif et aborde les soins de suivi, la bonne alimentation, l'activité physique, l'adaptation et l'ajustement, la survie et les ressources communautaires).
En Nouvelle-Écosse ont lieu des séances mensuelles sous le thème « La vie au-delà du cancer » (Living Beyond Cancer); elles sont animées par des infirmières, des radiothérapeutes et des infirmières en chimiothérapie et abordent, sous forme de présentations PowerPoint, des sujets tels que l'alimentation, les soins psychosociaux, le travail social et le bien-être spirituel. La présentation inclut des vidéos sur YouTube et le programme est accessible par télésanté pour les patients qui ne peuvent y assister en personne. Un autre programme provincial, celui-là intitulé «Sur le chemin du bien-être » (Your Way to Wellness), est conçu pour aider les patients à gérer la maladie chronique. De plus, deux fois par an, les patients sont invités à se joindre à un « groupe de transition des soins du cancer » (Cancer Transitions). Dans les cas où la transition des soins pourrait occasionner des difficultés aux patients (p. ex. de l'anxiété), le médecin ou l'intervenant-pivot peut les aiguiller vers le programme; les patients peuvent continuer de bénéficier du suivi de l'intervenant-pivot ainsi que de l'équipe de travail social. Cet atelier est conçu pour aider les patients à reprendre leur vie « normale » après la phase de traitement actif; le programme aborde une variété de sujets et dure entre 6 et 10 semaines. Les patients peuvent aussi avoir accès aux services de navigation à tout moment, de la suspicion de cancer à la phase de survie.

\section{DISCUSSION}

Étant donné le nombre croissant de survivants du cancer, tous ne pourront pas être suivis par des oncologues. Par conséquent, il faut transférer la responsabilité des soins de suivi aux médecins de première ligne lorsque le type de cancer s'y prête.

La recension des écrits a mis au jour des éléments révélateurs pour comprendre les obstacles au transfert exclusif des soins oncologiques vers le milieu communautaire, notamment le manque de clarté ou de définition des rôles, la difficulté à s'entendre sur la meilleure stratégie de suivi, les perspectives divergentes sur la responsabilité et la nature des soins, ainsi que le type d'information et le moment de la transmettre (Grunfeld et Earle, 2010; Cheung et al., 2013; Del Guidice, Grunfeld, Harvey, Piliotis et Verma, 2009; Haq et al., 2013; Mao et al., 2009; McCabe et Jacobs, 2012; Nissen et al., 2007). À l'heure actuelle, c'est à la jonction entre le rôle du médecin de première ligne et de l'oncologue que le processus achoppe. Le présent projet vise justement à produire des recommandations qui combleront ce fossé.

\section{Définition des rôles}

Selon Cheung et collègues (2013), lorsqu'il est question de cancers complexes, les médecins de première ligne ne possèdent peut-être pas les connaissances, la formation et l'expérience nécessaires pour gérer les problèmes et offrir des soins de suivi adéquats (notamment les examens de surveillance de la récidive), ce que confirment d'autres auteurs (Ackerman et al., 2014; Dawes et al., 2015; Del Guidice et al., 2009; Nissen et al., 2007; Salz et al., 2012). Toutefois, de nombreuses études réalisées au Canada et ailleurs dans le monde ont montré que le médecin de première ligne demeure le coordonnateur principal des soins pendant la période de survie et qu'il peut prodiguer efficacement les soins dans les cas de cancer à faible risque (Del Guidice et al., 2009; Mao et al., 2009; Grunfeld et al., 1995, 1996, 1999, 2006; Sisler, Brown et Stewart, 2004; Wattchow et al., 2006; Wood, 1993). 
Ye, Cheung, Goddard, Horvat et Olson (2015) ont établi que, règle générale, les radio-oncologues se chargent du suivi des patients aux cancers plus complexes (ceux qui touchent le système nerveux central, le tractus gastro-intestinal, la tête et le cou, et l'appareil gynécologique et génito-urinaire) à cause de la toxicité liée au traitement et de la possible nécessité de traitements supplémentaires. De l'avis des oncologues, les médecins de première ligne auraient besoin d'une formation plus poussée, ainsi que de lignes directrices plus détaillées, pour assurer le suivi ici. La recherche menée en NouvelleÉcosse confirme ce point de vue et note que les médecins de première ligne ne savent pas exactement à qui revient la responsabilité des différents aspects du suivi. En Alberta, ce sont souvent les infirmières praticiennes qui s'occupent des soins de suivi après les traitements oncologiques. Fait intéressant, la Saskatchewan insiste énormément sur la participation du fournisseur de soins primaires à toutes les étapes de la trajectoire du cancer; de plus, les patients qui n'ont pas confirmé être suivis par un médecin de première ligne ne reçoivent pas leur congé des soins oncologiques tant qu'ils n'ont pas remédié à la situation. En participant activement à toutes les étapes des soins, le médecin de première ligne renforce la confiance envers sa capacité d'assumer le suivi, puisqu'il est toujours au courant de la situation du patient (Urquart, Folkes, Babineau et Grunfeld, 2012). Au Manitoba, les patients ne reçoivent pas leur congé à moins d'avoir confirmé qu'ils étaient suivis par un médecin de première ligne et que ce médecin connaissait bien leur cas. Selon les données de Statistique Canada pour 2013, 15,5\% des Canadiens de 12 ans et plus n'avaient pas de médecin de première ligne (2014). Il s'agit là d'un point non négligeable dont les provinces doivent tenir compte au moment d'établir les modalités de transition des soins pour les patients qui n’ont pas de médecin de première ligne.

\section{Regards sur les soins}

Traditionnellement, la surveillance de la récidive du cancer est du domaine des oncologues; les médecins de première ligne se concentrent plutôt sur les interventions préventives associées aux troubles non cancéreux (Earle, Burstein, Winer et Weeks, 2003; Earle et Neville, 2004; Snyder et al., 2008). Sisler et collègues (2012) ont comparé la manière dont les survivants du cancer colorectal évaluaient la qualité des soins de suivi reçus après les traitements à l'évaluation qu'en faisaient les patients suivis par un médecin de première ligne. Résultats : les patients suivis par un médecin de première ligne accordaient une note positive à la transition des soins après le traitement actif, particulièrement lorsque le traitement n'avait entraîné aucune complication. Toutefois, les chercheurs ont noté qu'il aurait été bénéfique pour les patients à la convalescence plus difficile de recevoir davantage d'information et de services de soutien, ce qui aurait facilité leur transition. Le Manitoba utilise pour sa part un plan de soins différents pour les survivants du cancer comprenant l'avis de congé, les recommandations pour le suivi ainsi que le résumé du traitement personnalisé. L'avis de congé nomme clairement la personne chargée des soins de suivi (l'oncologue ou le médecin de première ligne), les prescriptions et les examens de routine à effectuer.
Pour assurer une transition sans heurt des soins oncologiques vers le milieu communautaire et permettre aux médecins de première ligne de surveiller efficacement la récidive au fil du temps, ces derniers doivent recevoir de la formation et du soutien. Urquhart et collègues (2012) ont observé que les survivants se sentent parfois dépassés par la transition du traitement actif vers le suivi de routine; leur responsabilité personnelle change et cela peut engendrer des sentiments de détresse et d'abandon. Les conclusions de l'étude de Del Guidice et collègues (2009) viennent confirmer cette observation; les patients pourraient s'attendre à être suivis par leur oncologue, ce qui inquiète les médecins de première ligne. Les patients sont contents que leur médecin participe aux soins de suivi, mais ils sont nombreux à vouloir être rassurés par leur oncologue et préfèrent être suivis par le spécialiste plutôt que par le médecin de première ligne. En outre, les patients craignent que ce dernier ne manque de connaissances et de compétences dans le domaine des soins du cancer, qu'il ne dispose pas du soutien adéquat ou qu'il n'ait pas accès à des spécialistes au besoin. Il est donc important de mieux former les intervenants de première ligne sur les enjeux liés à la survie au cancer et de les intégrer à la trajectoire des soins. Au bout du compte, les patients, les oncologues et les médecins de première ligne doivent tous avoir confiance en la capacité des médecins d'assumer pleinement les soins une fois le traitement oncologique terminé. En Saskatchewan, la participation essentielle des médecins de première ligne à toutes les étapes de la maladie contribue grandement à accroître la confiance du patient et de l'oncologue envers la capacité des médecins de première ligne à assurer le suivi une fois le traitement terminé.

\section{Type d'information et moment choisi pour la transmettre}

Le type d'information et le moment où elle est remise aux patients et aux médecins de première ligne constitue un aspect important de la transition des soins, particulièrement pour les médecins de première ligne; les pratiques en vigueur en Colombie-Britannique et la littérature sur le sujet dénotent pourtant des lacunes dans ce domaine (Del Guidice et al., 2009; Haq et al., 2013). Le type d'information transmise aux patients varie énormément d'un bout à l'autre du pays et aucune province ne dispose réellement de normes en la matière. Dans la plupart des provinces, les cliniques de soins du cancer possèdent toutes leur propre bagage d'information, que ce soit sous forme de bibliothèque, de brochures et de feuillets sur papier ou de documents en ligne. Cette information couvre différents enjeux liés à la survie au cancer (p. ex. les effets secondaires ou persistants possibles, une bonne alimentation, un mode de vie sain, la médication, ainsi que les séquelles psychosociales et émotionnelles).

Pour que le médecin de première ligne puisse discuter de la phase post-traitement avec le patient en toute connaissance de cause, il est crucial qu'il reçoive les renseignements sur le patient au bon moment (c'est-à-dire dès que le patient quitte le milieu oncologique) (Haq et al., 2013). En général, dans toutes les provinces, on tente de remettre l'information (sous forme d'avis dicté, d'avis de transfert des soins ou de plan de soins) au médecin de première ligne au moment du congé. 
Les aide-mémoire à l'intention des patients et des médecins de famille, le rôle de coordonnateur de la transition des soins, le rendez-vous de transition et le plan de soins pour les survivants (qui doit comprendre les consignes des spécialistes, des lignes directrices claires et personnalisées orientant le suivi, un accès rapide aux examens de dépistage et un réacheminement vers les soins spécialisés en temps opportun) permettent tous de transmettre l'information et de faciliter la transition des patients une fois qu'ils ont reçu leur congé. (Del Guidice et al., 2009; Smith, Singh-Carlson, Downie, Payeur et Wai, 2011; Ye, Cheung, Goddard, Horvat et Olson, 2015).

\section{Recommandations}

Pour améliorer les pratiques actuelles de transition des soins $\mathrm{du}$ cancer en Colombie-Britannique et dans l'ensemble du Canada, et ainsi maintenir la qualité des soins, des changements s'imposent. La présente étude exploratoire a permis de cerner certaines lacunes, notamment dans la définition des rôles, la variabilité des professionnels en charge des soins, ainsi que les besoins et les exigences en matière d'information. Quatre recommandations sont ressorties de notre analyse : 1) l'adoption d'un aide-mémoire pour le suivi; 2) la mise en place de rendez-vous de transition; 3) la création d'un poste de coordonnateur de la transition des soins; 4) l'utilisation d'un plan de soins pour survivants. Les rendez-vous de transition et la création d'un poste coordonnateur de la transition des soins peuvent être les premiers jalons d'une démarche visant à combler les lacunes dans le processus de transition. De leur côté, l'aide-mémoire pour le suivi et le plan de soins pour survivants peuvent répondre aux besoins d'information, un aspect primordial pour transférer les soins en toute confiance aux médecins de première ligne.

1. Le rendez-vous de transition est un mécanisme efficace pour faire le pont entre les soins oncologiques et les soins communautaires et peut servir de point de contact entre les oncologues et les médecins de première ligne. Ce rendez-vous contribue à la continuité des soins et permet de répondre aux besoins des patients et de les faire participer à leurs propres soins. Le rendez-vous de transition est souvent mené par une infirmière praticienne; ainsi, les soins de suivi ne seront plus le seul apanage de l'oncologue et pourront être confiés progressivement et entièrement aux médecins de première ligne. La Saskatchewan, l'Ontario, l'Île-du-Prince-Édouard, la Nouvelle-Écosse et Terre-Neuve offrent aux patients un atelier ou un programme pendant la période de transition ou alors des séances d'information à l'intention des survivants. En présentant aux patients des renseignements de base sur la survie au cancer, ces ateliers leur donnent les moyens de mieux comprendre les soins qu'ils reçoivent et d'y prendre une part active, contribuant ainsi à combler le fossé existant entre l'oncologie et le milieu communautaire.

2. Le coordonnateur de la transition des soins joue un peu le même rôle que le rendez-vous de transition, mais en agissant comme personne-ressource en lien direct avec le patient. Il donne au patient de l'information sur les programmes communautaires et les ressources disponibles dans sa région ou son secteur pour l'aider à gérer lui-même ses soins. Le coordonnateur de la transition des soins devient un point de contact unique pour le patient; il est toujours là pour l'aider pendant la phase de survie. La création d'un poste de coordonnateur de la transition des soins est une étape importante pour établir le lien entre l'oncologue et le médecin de première ligne. Le coordonnateur peut servir de relais dans la continuité des soins pour veiller à ce que les besoins des patients soient satisfaits et pour encourager les patients à participer activement à leurs soins.

3. Les plans de soins pour les survivants sont utilisés pour améliorer la communication entre l'oncologue, le médecin de première ligne et le patient (Ganz, Casillas et Hahn, 2008; Naidoo et al., 2013). Le Manitoba, l'Ontario, la NouvelleÉcosse et Terre-Neuve ont déjà adopté, sans les standardiser, des plans de soins adaptés aux survivants sous une forme ou sous une autre. L'avis de congé ou de transfert des soins que la plupart des provinces utilisent en guise d'outil de communication contient souvent certains éléments du plan de soins; ils permettent donc d'améliorer la communication et de réduire l'écart entre l'oncologue traitant et le médecin de première ligne. L'information qui figure dans le résumé du traitement est similaire entre le Manitoba, l'Ontario et Terre-Neuve; on y précise certains éléments clés tels que le profil démographique du patient, l'information concernant l'équipe de soins, la chirurgie (date et détails), l'analyse pathologique de la tumeur (stade, grade), le traitement reçu et les détails de celui-ci (chimiothérapie, radiothérapie, hormonothérapie), les effets secondaires anticipés (aigus et chroniques) et les renseignements sur les examens diagnostics (par exemple, la date de la dernière mammographie et la date prévue de la prochaine pour les patientes atteintes de cancer du sein). Le plan de suivi précise les signes et symptômes de récidive, ainsi que les examens recommandés et l'horaire des rendez-vous.

\section{CONCLUSION}

La survie au cancer est un sujet complexe qui nécessite communication et coordination entre le milieu de l'oncologie et le milieu communautaire pour assurer aux survivants une transition des soins sans heurt. Le présent projet a mis au jour les différences entre les pratiques de transition des soins des différentes provinces et proposé quelques recommandations réalistes pour augmenter la qualité des soins du cancer. L'évaluation des pratiques n’a pas révélé de grandes surprises; la majeure partie des renseignements fournis en entrevue sont simplement venus confirmer les données de la littérature sur le sujet. La plupart des participants aux entrevues étaient manifestement conscients des exigences et des besoins croissants en matière de soins de qualité pour le suivi du cancer, et les lacunes dans les programmes et les mesures de soutien pour les survivants étaient généralement attribuées à un manque de fonds et de temps. Plusieurs entrevues se terminaient sur des mots du type "J'aimerais qu'on puisse en faire plus » ou « Notre système actuel n'en fait pas assez, mais nous travaillons à l'améliorer ». Voici quelques initiatives qu'il serait utile d'explorer : 1) organiser des séances d'information à 
l'intention des médecins de première ligne pour leur expliquer toute la difficulté d'offrir des soins de suivi adéquats ou 2) créer un programme ou une clinique pour les survivants dont l'un des volets serait le suivi des résultats de santé.

\section{REMERCIEMENTS}

Je désire remercier de sa générosité le programme de survie au cancer et de soins primaires (Survivorship and Primary Care

\section{RÉFÉRENCES}

Ackerman, S., Gleaon, N., Monacelli, J., Collado, D., Wang, M., Ho, C., ... Gonzales, R. (2014). When to repatriate? Clinicians' perspectives on the transfer of patient management from specialty to primary care. Journal of General Internal Medicine, 29(10), 1355-1361.

British Columbia Cancer Agency (BCCA). (2015). Survivorship Q primary care program. Retrieved from http://www. bccancer.bc.ca/health-professionals/professional-resources/ survivorship-primary-care

Canadian Cancer Society. (2015). Cancer statistics at a glance. Retrieved from http://www.cancer.ca/en/cancer-information/cancer-101/ cancer-statistics-at-a-glance/?region $=$ on

Cheung, W., Aziz, N., Noone, A., Rowland, J., Potosky, A., Ayanian, J., ... Earle, C. (2013). Physician preferences and attitudes regarding different models of cancer survivorship care: A comparison of primary care providers and oncologists. Journal of Cancer Survivorship, 7, 343-354.

Dawes, A., Hemmelgarn, M., Nguyen, D., Sacks, G., Clayton, S., Cope, J., ... Maggard-Gibbons, M. (2015). Are primary care providers prepared to care for survivors of breast cancer in the safety net? Cancer, 121(8), 1249-1259.

Del Guidice, M., Grunfeld, E., Harvey, B., Piliotis, E., \& Verma, S. (2009). Primary care physicians' views of routine follow-up care of cancer survivors. Journal of Clinical Oncology, 27(20), 3338-3345.

Earle, C., Burstein, H., Winer, E., \& Weeks, J. (2003). Quality of nonbreast cancer health maintenance among elderly breast cancer survivors. Journal of Clinical Oncology, 21(8), 1447-1451.

Earle, C. \& Neville, B. (2004). Underuse of necessary care among elderly colorectal cancer survivors. Cancer, 101(8), 1712-1719.

Fereday, J., \& Muir-Cochrane, E. (2006). Demonstrating rigour using thematic analysis: A hybrid approach of inductive and deductive coding and theme development. International Journal of Qualitative Methods, 5(1), 80-92.

Ganz, P., Casillas, J., \& Hahn, E. (2008). Ensuring quality care for cancer survivors: Implementing the survivorship care plan. Seminars in Oncology Nursing, 24(3), 208-217.

Government of Canada. (2014). Statistics Canada: Health facts sheet. Ottawa: Statistics Canada.

Grunfeld, E. (2006). Looking beyond survival: How are we looking at survivorship. Journal of Clinical Oncology, 24(32), 5166-5169.

Grunfeld, E., \& Earle, C. (2010). The interface between primary and oncology specialty care: Treatment through survivorship. Journal of National Cancer Institute Monographs, 40, 25-30.

Grunfeld, E., Gray, A., Mant, D., Yudkin, P., Adewuyi-Dalton, R., Coyle, D., et al. (1999). Follow-up of breast cancer in primary care vs specialists care: Results of an economic evaluation. British Journal of Cancer, 79(7/8), 1227-1233.

Grunfeld, E., Levine, M., Julian, J., Coyle, D., Szechtman, B., Mirsky, D., et al. (2006). Randomized control trial of long-term follow-up for early-stage breast cancer: A comparison of family physician versus specialist care. Journal of Clinical Oncology, 24(6), 848-855.

Grunfeld, E., Mant, D., Yudkin, P., Adewuyi-Dalton, R., Cole, D., Stewart, J., et al. (1996). Routine follow-up of breast cancer
Program) de la British Columbia Cancer Agency, qui m'a permis d'inscrire mes recherches dans son vaste projet, ainsi que Sue Willis, ma directrice de thèse à l'Université Sheffield Hallam, pour toute l'aide et le soutien qu'elle m'a apportés.

\section{CONFLIT D'INTÉRÊTS}

L'auteure déclare l'absence de tout conflit d'intérêts.

in primary care: Randomized control trial. British Medical Journal, 313(7058), 665-669.

Grunfeld, E., Yudkin, P., Adewuyi-Dalton, R., Vessey, M., \& Mant, D. (1995). Follow-up in breast cancer. Quality of life unaffected by general practice follow-up. British Medical Journal, 11(6996), 54.

Hagan, T., \& Donovan, H. (2012). Self-advocacy and cancer: A concept analysis. Journal of Advanced Nursing, 69, 2348-2359.

Haq, R., Baker, N., Dastur, D., Leung, F., Leung, E., Li, B., ... Parsons, J. (2013). Designing a multifaceted survivorship care plan to meet the information and communication needs of breast cancer patients and their family physicians: Results of a qualitative pilot study. Medical Informatics and Decision Making, 13(76), 1-13.

Mao, J., Bowman, M., Stricker, C.T., DeMichele, A., Jacobs, L., Chan, D., \& Armstrong, K. (2009). Delivery of survivorship care by primary care physicians: The perspective of breast cancer patients. Journal of Clinical Oncology, 27, 933-938.

McCabe, M., \& Jacobs, L. (2012). Clinical update: Survivorship caremodels and programs. Seminars in Oncology Nursing, 28(3), e1-e8.

Naidoo, J., Hayes, E., Teo, M., Morgan, A., Calvert, P., \& O’Connor, M. (2013). An Irish breast cancer survivorship study: Are we meeting our patient's needs? Irish Medical Journal, 106(9), 264-266.

Nissen, M., Beran, M., Lee, M., Mehta, S., Pine, D., \& Swenson, K. (2007). Views of primary care providers on follow-up care of cancer patients. Family Medicine, 39, 477-482.

Salz, T., Oeffinger, K.C., Lewis, P.R., Williams, R.L., Rhyne, R.L., \& Yeazel, M.W. (2012). Primary care providers' needs and preferences for information about colorectal cancer survivorship care. Journal of the American Board of Family Medicine, 25, 635-651.

Sisler, J., Brown, J., \& Stewart, M. (2004). Family physicians' roles in cancer care: Survey of patients on a provincial cancer registry. Canadian Family Physician, 50, 889-896.

Smith, S., Singh-Carlson, S., Downie, L., Payeur, N., \& Wai, E. (2011). Survivors of breast cancer: Patient perspectives on survivorship care planning. Journal of Cancer Survivorship, 5, 337-344.

Snyder, C., Earle, C., Herbert, R., Neville, B., Blackford, A., \& Frick, K. (2008). Preventative care for colorectal cancer survivors: A 5-year longitudinal study. Journal of Clinical Oncology, 26(7), 1073-1079.

Urquhart, R., Folkes, A., Babineau, J., \& Grunfeld, E. (2012). Views of breast and colorectal cancer survivors on their routine follow-up care. Current Oncology, 19(6), 294-301.

Wattchow, D., Weller, D., Esterman, A., Pilotto, L., McGorm, K., Hammett, Z., ... Silagy, C. (2006). General practice vs surgicalbased follow-up for patients with colon cancer: Randomised controlled trial. British Journal of Cancer, 94(8), 1116-1121.

Wiljer, D., Urowitz, S., Jones, J., Kornblum, A., Secord, S., \& Catton, P. (2013). Exploring the use of the survivorship consult in providing survivorship care. Support Care Cancer, 21, 2117-2124.

Wood, M.L. (1993). Communication between cancer specialists and family doctors. Canadian Family Physician, 39, 49-57.

Ye, A., Cheung, W., Goddard, K., Horvat, D., \& Olson, R. (2015). Follow-up patterns of cancer survivors: A survey of Canadian radiation oncologists. Journal of Cancer Survivorship, 9, 388-403. 